\title{
ANALYSIS OF ROCK DYNAMIC STRESSES DURING THE DRILLING BY POLYCRYSTALLINE DIAMOND COMPACT BITS
}

\author{
Yu, J. P.*; Zou, D. Y.* \& Zhang, Y.** \\ *School of Petroleum Engineering, China University of Petroleum (East China), Qingdao, 266580, \\ China \\ ** Department of Petroleum Engineering, University of Alaska Fairbanks, Fairbanks, Alaska, 99775, \\ United States of America \\ E-Mail: eugene_yujp@126.com
}

\begin{abstract}
In view of shortening the development period of polycrystalline diamond compact (PDC) bits, the finite element method was adopted to simulate the dynamic stress of rocks. By employing drilling related theories, the three dynamic principal stresses of rock were analysed and the dynamic rock-breaking criterion was established. Second, the drilling model of PDC core bit was constructed, and the stress was simulated and calculated. Finally, laboratory tests were carried out to verify the simulation results. The analytical results demonstrate that the two obvious stages in the rock-breaking process are the initial rock-breaking stage and the normal one. The dynamic rock-breaking stress in the normal drilling stage varies from 66.3 to $99.6 \mathrm{MPa}$, which is lower than $278.4 \mathrm{MPa}$ in the initial rock-breaking stage. During spud drilling, the axial force and the tangential force are 1.85 and $1.60 \mathrm{kN}$, respectively. During normal drilling, the axial force ranges from 0.2 to $0.9 \mathrm{kN}$, and the tangential force from 0.15 to $0.6 \mathrm{kN}$. The load of normal drilling is lower than the spudding load, and the bit is more likely to be damaged during spudding. The bit is normally worn during normal drilling.

(Received in August 2020, accepted in November 2020. This paper was with the authors 1 month for 1 revision.)
\end{abstract}

Key Words: PDC Bit, Rock Breaking, Dynamic Stress, Simulation Calculation, ANSYS/LS-DYNA, Wear Resistance

\section{INTRODUCTION}

Polycrystalline diamond compact (PDC), with its merits of good hardness, impact resistance, and wear resistance, is considered the most ideal material for the cutting teeth of drill bits in the petroleum industry. In engineering practice, PDC bits have been proven to have high drilling speed, low bit pressure, long service life, stable performance and high rock-breaking efficiency, and it is widely used in oil-gas drilling [1]. Considering the excellent performance of PDC materials, experts and scholars have designed PDC bits with specific characteristics depending on different formation rock features.

However, with the expansion of drilling areas, higher requirements are raised for the performance of PDC bits. A variety of influencing factors should be considered in improving the performance of PDC bits suitable for certain stratum and drilling technology and designing special types of bits. This higher-level requirement presents a huge problem in the bit design and production industry.

Scholars attempted to analyse the whole development and production process of bits and investigated rock-breaking principles via simulation and testing [2-4]. The wear resistance, mechanical properties, and impact resistance of bits were simulated and evaluated. Furthermore, the rock-breaking effect was determined by simulating the drilling process. Thus, knowing how to shorten the design cycle, decrease the cost of R\&D and testing, reduce the links of "multiple models, tests, and optimizations," and design rapidly the special bits are urgent research concerns. 
This study determined the dynamic rock-breaking criteria of controlling rock-breaking conditions. Moreover, the rock-breaking process was simulated by means of the finite element method (FEM) [5-7], and the position relationship between the blade and the cutter was analysed. The dynamic rock-breaking stress of bits was analysed to evaluate the rock-breaking efficiency and wear resistance. The proposed method aims to establish a drilling model and repeatedly analyse dynamic rock-breaking stress by using different models. The results of this study offer important reference value and engineering significance in terms of shortening the development period and enhancing drilling efficiency of PDC bit.

\section{STATE OF THE ART}

Scholars at present have studied PDC bits suitable for various formations from different perspectives [8-18]. Miyazaki et al. [8] investigated the performance of a PDC bit for different rocks and the relationship between wear resistance and drilling parameters via laboratory testing and proposed the efficient use of PDC bits. However, their study did not involve the design and evaluation of PDC bits. Mazen et al. [9] proposed a new mathematical model to predict PDC bit performance, investigated the influences of constructure, hydraulics, and rock strength on PDC bit performance, and predicted bit wear. Although they attempted to relate the drilling parameters to bit performance and wear, their findings mainly focused on bit wear. Agostini and Sampaio [10], who used the Bayesian probabilistic neural network, proposed a method of monitoring the wear of PDC bits in a hard rock layer of an ultra-deep well in real time to prolong the service life of bits, but they did not conduct a rock-breaking simulation of it. Abbas and Musa [11] reported that specific PDC bits were needed for different formations, and the performance could be evaluated according to wear resistance. Additionally, the rationality of analysing the wear performance by using Raman shift and Fourier transform infrared spectroscopy was proved. They also evaluated the performance of engineering bits instead of that of a new bit. Agawani et al. [12] introduced a multifunctional bit by combining PDC and tungsten carbide insert, which is applicable to heterogeneous carbonate formations owing to good impact resistance characteristic and found that the rate of penetration (ROP) was twice that of a conventional drill bit. However, they failed to introduce a design and evaluation process for $t$ bit. Abbas [13] reviewed the literature about bit wear and used finite element analysis and the discrete element method to simulate and calculate bit wear. The author also studied PDC bits from the perspective of service life but focused on application rather than design. Saksala et al. [14] numerical simulated and experimentally studied an impact drill and dynamically simulated rock breaking based on a bit-rock interaction model. Their study emphasized the relationship between impact velocity and rock breaking, but bit design and evaluation were not implemented. Wang et al. [15] experimentally studied the optimal tooth arrangement mode of a PDC bit suitable for granite and hard sandstone and examined the relationship between tooth arrangement mode and service life and ROP. Although the designed bit showed strong pertinence, the design and moulding process was long and complex, suggesting a weakness in meeting the engineering requirements of rapid bit design. Wang et al. [16] established a three-dimensional dynamic rock-breaking model by using a threedimensional finite element software to measure the mechanical properties of a rock and subsequently studied the rock-breaking rule. Nevertheless, they failed to evaluate the performance of the bit because of the bit-rock coupling. Zou et al. [17] designed a special PDC bit for a special stratum and obtained the power function law of rock-breaking efficiency via experimentation. The bit design and moulding process derived in their work was the same as that of Wang et al. [15]. Despite the focus on personalized design, they did not propose a method to shorten the personalized design cycle. Niu et al. [18] analysed the structural characteristics 
and rock-breaking mechanism of a bit and evaluated the service life of a PDC bit via experimentation but failed to propose a new design method.

The abovementioned past studies mainly concentrated on the service performance, mechanical properties, and wear resistance of PDC bits, but the bit design process entailing simulation was seldom considered, especially in terms of designing bits using a computer program to shorten its design cycle by means of simulating the process of drilling rock breaking. Consequently, the present study simulated the drilling process to analyse the dynamic stress of rock breaking. A drilling model was established on the basis of a dynamic rock-breaking criterion, and drilling parameters were employed to calculate the dynamic stress of rocks by using different PDC bits. The proposed method can lay a foundation for bit structure design and bit performance and wear resistance evaluation. The simulation was implemented on a computer instead of using a production model for laboratory testing. In this manner, the intermediate links of bit design could be reduced and the design cycle of bits could be shortened.

The remainder of this study is organized as follows. Section 3 describes the dynamic rockbreaking criterion for controlling rock breaking and presents the constructed drilling model used to calculate dynamic stress when the rock is breaking with a PDC bit. Section 4 presents the FEM adopted for the structural analysis to calculate the dynamic stress of broken rocks and analyse the rock-breaking process. The variation characteristics of the dynamic stress of broken rocks over time during drilling and the influence of the position relationship between the bit blade and cutter on rock-breaking efficiency are also discussed. Section 5 summarizes the current study and draws the relevant conclusions.

\section{METHODOLOGY}

The dynamic stress of a breaking rock was analysed via simulation and calculation to determine the performance and wear resistance of different PDC bits. The method is described in the succeeding subsections.

\subsection{Dynamic rock-breaking criteria}

Drilling is an interaction process between a bit and a rock, in which the rock is broken by bit. The chosen appreciate theory plays an important role in simulating and calculating rock breaking. Rocks are a brittle material. The commonly used rock-breaking criteria in the petroleum engineering field include the von Mises, Mohr-Coulomb, and Mogi-Coulomb criterion [19]. These criteria have the commonality of being applicable only to rock breaking under static load. The criteria should be properly adjusted prior simulating and calculating dynamic conditions. The stress working conditions of the rock-breaking were considered in this study, and a rock-breaking criterion during the drilling of a six-cutter core PDC bit was established by employing relevant basic theories.

The structural diagram of the six-cutter core PDC bit is shown in Fig. 1, and the corresponding simulation calculation model is presented in Fig. 2.

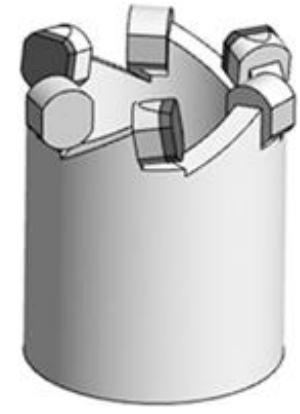

Figure 1: Structural diagram of a six-cutter core PDC bit.

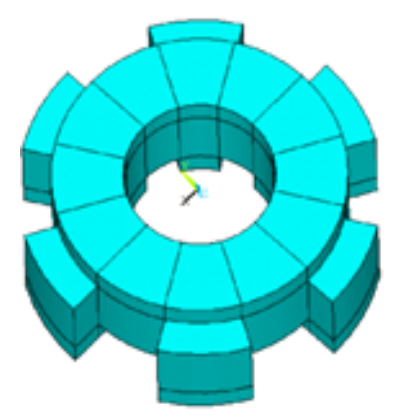

Figure 2: Model of bit. 
In an actual drilling process in which an infinite rock is drilled, the hollow position of the drill bit is considered the rock core. In a rock-breaking process, the drill bit breaks the annular rock below it. The annular micro-segment (AMS) below the drill bit was analysed in this study. The schematic depicting the stress analysis is shown in Fig. 3.

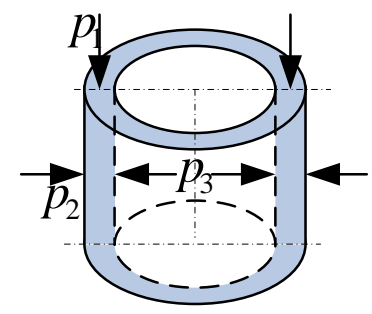

Figure 3: Pressure of an AMS.

As the inside of a rock is cylindrical and the outside part is huge, the WOB on top of the rock acts on the annular rock. Rocks inside and outside the ring can be regarded the rigid bodies. Surface pressure $p_{1}$ is generated on top of the annular micro-segment by WOB. According to elasticity theory, the external rock generates confining pressure $p_{2}$ on the AMS under the condition that external and internal rocks are regarded the rigid constraints, and the internal rock generates internal pressure $p_{3}$ on the AMS (Fig. 3). The stress state of the element by WOB inside the AMS was analysed in this work (Fig. 4 a). Under the action of the three pressures, the three normal stresses acting on the unit body are able to meet the following conditions:

where:

$$
\left.\begin{array}{l}
\sigma_{z}=f(P, S) \\
\sigma_{r}=\sigma_{t}=\frac{\mu}{1-\mu} \sigma_{z}
\end{array}\right\}
$$

- $\sigma_{z}$ is normal stress (MPa) at a certain point on the ring in the direction of WOB. Pressure stress, which is positive, is a function of WOB $P$ and bit structure parameter $S$.

- $\sigma_{r}$ and $\sigma_{t}$ represent normal stress (MPa) at the point in two other directions (circumferential and radial directions). Pressure stress, which is positive, is a function of $\sigma_{z}$, and rock properties.

- $\mu$ is the Poisson's ratio of the rock.

During the drilling process, the bit moves constantly and has a cutting effect on the rock (i.e., related to the effect of the torque on the bit). Shear stress $\tau$ is generated on the rock contacting with the bit teeth, and the direction of the shear stress is consistent with the rotation direction of the bit. Therefore, shear stress $\tau$ acts on the stress element surface where $\sigma_{z}$ and $\sigma_{t}$ are loaded. The unit entails the four stress components of $\sigma_{r}, \sigma_{t}, \sigma_{z}$ and $\tau$ shown in Fig. $4 \mathrm{~b}$.

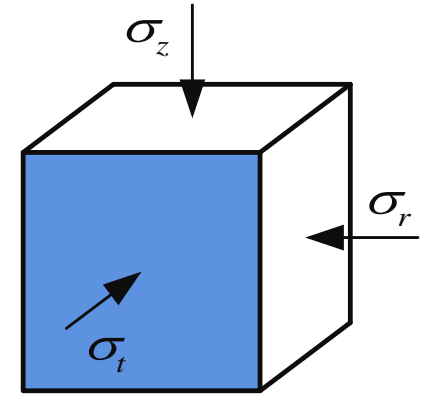

a)

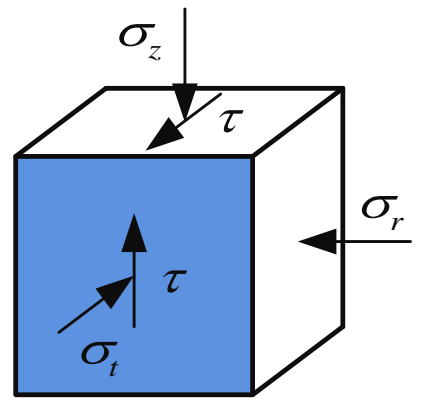

b)

Figure 4: Element's stress state.

The three dynamic principal stresses [20] on the rock are calculated as follows: 
where:

$$
\left.\begin{array}{l}
\sigma_{d}^{3}-J_{1} \sigma_{d}^{2}+J_{2} \sigma_{d}-J_{3}=0 \\
J_{1}=-\left(\sigma_{r}+\sigma_{t}+\sigma_{z}\right) \\
J_{2}=\sigma_{r} \sigma_{t}+\sigma_{t} \sigma_{z}+\sigma_{z} \sigma_{r}-\tau^{2} \\
J_{3}=\left(\sigma_{t} \sigma_{z}-\tau^{2}\right) \sigma_{r}
\end{array}\right\}
$$

- $\sigma_{d}$ denotes the dynamic principal stress during drilling (MPa);

- $J_{1}, J_{2}$ and $J_{3}$ represent the first, second, and third stress tensor invariants; and $\sigma_{d 1}, \sigma_{d 2}$ and $\sigma_{d 3}$ are three principal stresses during drilling $(\mathrm{MPa})$.

On the basis of Eq. (2), three dynamic principal stresses can be obtained. When the maximum stress among three principal stresses is greater than the tensile fracture limit of the rock, the rock will be broken. This phenomenon accords with the strength theory. The three principal stresses are dynamic principal stresses comprising WOB, bit structure parameters, bit torque, friction coefficient between bit and rock, and rock properties. The factors influencing rock breaking can thus be determined. Different from the von Mises, Mohr-Coulomb, and Mogi-Coulomb criterion that only comprise static indexes, the rock-breaking criterion proposed in this study can reflect the actual rock-breaking process. Moreover, the rock-breaking criterion conforms with actual drilling conditions.

Here, the dynamic rock-breaking criterion corresponds to the control condition for simulating rock breaking.

\subsection{Drilling model and gridding of the six-cutter core PDC bit}

Although the dynamic stress of rock breaking can be analysed according to Eqs. (1) and (2), the application of these formulas is limited by the randomness, complexity, and heavy calculation burden of drilling. Therefore, simulation calculation is commonly used in engineering. The ANSYS/LS-DYNA calculation software is an effective tool for analysing nonlinear contact problems during drilling.

First, a bit model and a rock model were constructed (Fig. 2) according to Macyszyn method [21]. A $0.0001 \mathrm{~m}$ gap was reserved at the bottom surface where the bit model and the rock model come in contact with each other, i.e., between the bit and the rock, to increase the number of contact units. The rock-breaking drilling model is shown in Fig. 5.

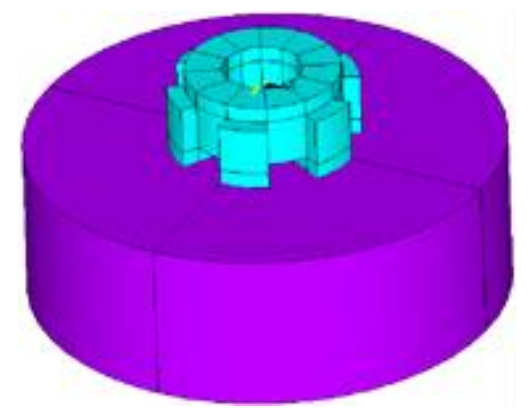

Figure 5: Drilling model.

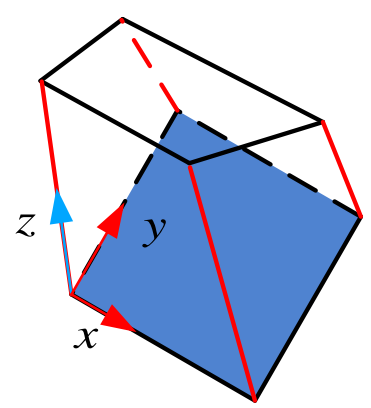

Figure 6: Hex. elements and coordinates.

According to relevant data $[9-18,22]$ and Saint-Venant principle, the radial dimension of the rock calculated by the simulation should be more than three times that of the bit to meet the accuracy analysis requirement. Moreover, the axial height of the rock should be more than twice the size of the bit model. According to this guiding principle, a $\Phi 600 \mathrm{~mm} \times 200 \mathrm{~mm}$ cylindrical rock model was established. Rock breaking corresponds to the contact between the rock and the bit. Thus, rock breaking involves nonlinear problem and requires a set of rock-breaking criteria. SOLID164, a solid unit commonly used in LS-DYNA, was employed in this work to 
establish the model and divide the units. SOLID164 is an explicit solid unit structure consisting of eight nodes, allowing the freedom of translation, velocity, and acceleration in the $\mathrm{x}, \mathrm{y}$, and $\mathrm{z}$ directions, as shown in Fig. 6.

In the rock-breaking process, the rock breaking is influenced not only by the load exerted by the drilling and the geometric model of the bit but also the rock's mechanical parameters, such as water content, composition, particle size, and porosity, and whether the rock contains cracks. Rock is a nonlinear material and undergoes not only elastic deformation but also complex reconfiguration, such as plastic deformation and brittle fracture. Therefore, an appropriate material model is needed to accurately simulate the mechanical response of rock breaking. The material model proposed by Holmquist, Johnson and Cook (HJC), aptly called the HJC material model, is a brittle material damage model suitable for large-strain, high-strain rate, and high-pressure conditions. Consequently, this study used the HJC model to simulate rock breaking during drilling [23].

For the grid division of bit, the annulus grids were uniformly divided, and the grids in the axial direction were uniformly divided by region, with the dense grids located near the rock and the uniformly distributed grids located far away from the rock. The method of dividing a rock as a grid is simple. The contact part with the bit and the central part of the rock were refined, and the contact with the bit was realized on the basis of the contact condition between the rock and the bit. The contact between the rock and the bit was set as the erosion contact in the surface-to-surface contact. The static friction coefficient between the rock and the bit was 0.35 , and the dynamic friction coefficient was 0.3. Except for the contact surface, all points on the outer surface of rock were completely constrained. The grid division is presented in Fig. 7.

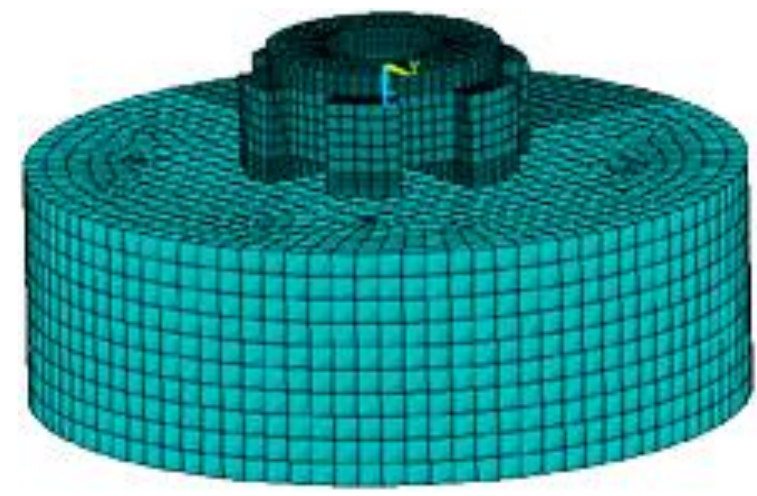

Figure 7: Grid division of the drilling model.

The bit drilled around the central axis and the rock-breaking condition were controlled according to the criteria given in Section 3.1. The drilling speed was $0.005 \mathrm{~m} / \mathrm{s}$ and rotational speed loads was $9 \mathrm{rad} / \mathrm{s}$. LS-PREPOST, a special post-processor for the LS-DYNA software, was employed for post-processing to obtain the nephogram of the change in dynamic stress and the expansion diagram of the breaking pit in the breaking process.

This process is simple that it can be completed using a computer. And the bit chosen that could satisfy the requirements of the formation rock properties is applied in engineering to complete engineering-type tests. Overall, the method adopted in this work is fairly simple.

\subsection{Laboratory verification test}

The test system of the $\mathrm{XY}-2 \mathrm{~B}$ drilling rig in Fig. 8 is its own test system, which is an integral part of XY-2B drilling rig. The system can collect WOB, torque, footage, and time data in real time. The test accuracy of the system is as follows: WOB (axial force) of $0.0001 \mathrm{kN}$, torque (tangential force) of $0.0001 \mathrm{kN}$, and footage of $0.0001 \mathrm{~mm}$. The accuracy meets the requirement of the drilling parameter test. 


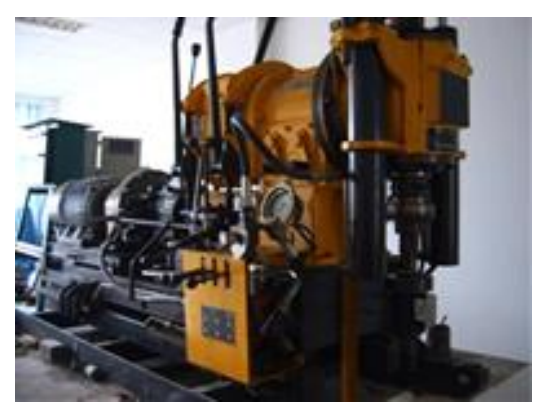

Figure 8: XY-2B drilling rig.

\section{RESULT ANALYSIS AND DISCUSSION}

\subsection{Analysis of dynamic stress and breaking depth of the simulated drilling rock-breaking}

The property and loading parameters of the rock was inputted into the system. Then, the maximum dynamic stress at different times and the rock-breaking depth $h$ at the corresponding position is given in Table I.

Table I: Relationship among $\sigma_{d}, h$ and time during the rock-breaking process.

\begin{tabular}{|l|c|c|c|c|c|c|c|c|}
\hline$t(\mathrm{~s})$ & 0.02 & 0.04 & 0.06 & 0.07 & 0.1 & 0.35 & 0.6 & 0.86 \\
\hline$\sigma_{d}(\mathrm{MPa})$ & 174.1 & 235.4 & 274.0 & 278.4 & 66.3 & 85.0 & 70.8 & 99.6 \\
\hline$h(\mathrm{~mm})$ & & & & 0.35 & 0.29 & 0.31 & 0.30 & 0.33 \\
\hline
\end{tabular}

As shown in Table I, the dynamic stress of the rock increased sharply from 0 to $278.4 \mathrm{MPa}$ at $0.07 \mathrm{~s}$ of the drilling simulation, but the rock-breaking depth was not given, indicating that the rock was not broken before $0.07 \mathrm{~s}$. Beginning $0.07 \mathrm{~s}$ of the drilling simulation, the rockbreaking depth fluctuated between 0.29 and $0.35 \mathrm{~mm}$. The rock-breaking process data presented in Table I can be divided into two stages, namely, the initial rock-breaking stage and the normal rock-breaking stage. In the initial rock-breaking stage, the rock was broken under the limit value of the dynamic stress. In the normal drilling rock-breaking stage, the rock started to break during the normal drilling, although the dynamic stress value of the rock was stable in the lower dynamic stress range.

The maximum dynamic stress occurred at $0.07 \mathrm{~s}$ (Table I), while the maximum axial force and tangential force occurred between 0.07 and $0.08 \mathrm{~s}$ (Fig. 9). Then, the dynamic stress sharply decreased to 66.3-99.6 MPa. The changes were relatively stable, as verified by the experiments.

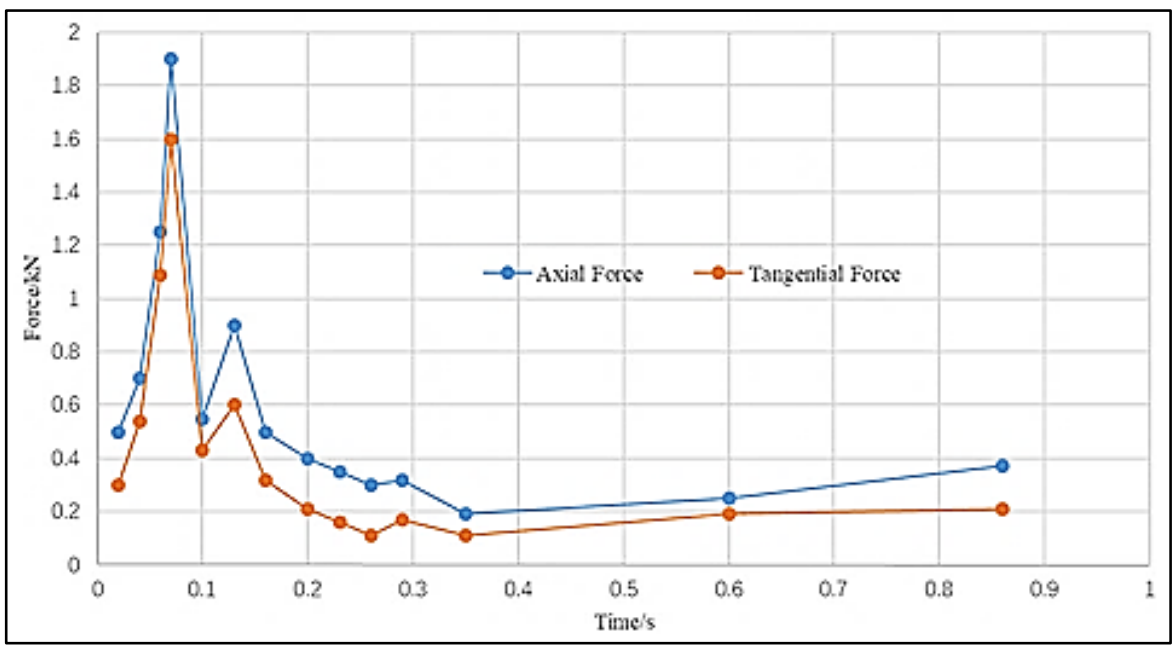

Figure 9: Change in rock-breaking load of the PDC bit over time. 


\subsection{Analysis of dynamic stress at the initial rock-breaking stage}

The dynamic stress of the rock changed with time (Table I). The dynamic stress was $174.1 \mathrm{MPa}$ when the drilling reached for $0.02 \mathrm{~s}$ and increased to $278.4 \mathrm{MPa}$ at $0.07 \mathrm{~s}$ shown in Fig. 10. The stress at the contact part between the rock and the bit was the largest, followed by the stress between the bit blades. The analysis of the rock-breaking process is given in Section 4.4.

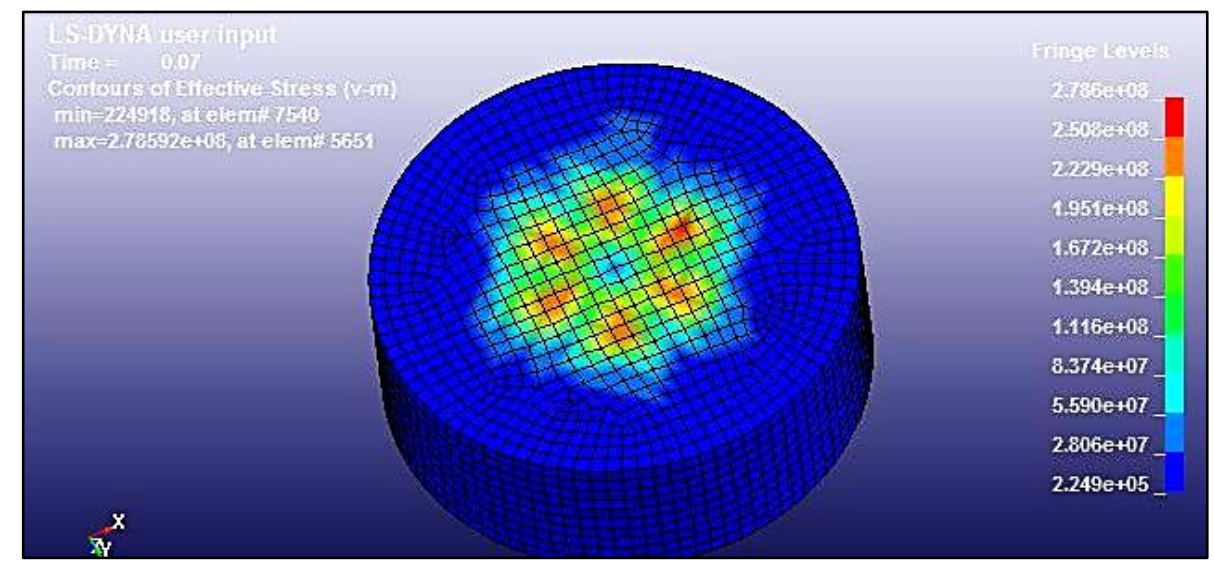

Figure 10: Maximum stress before rock breaking at $0.07 \mathrm{~s}$.

The simulation results indicate that the dynamic stress is at the maximum when the drilling reached $0.07 \mathrm{~s}$, and the rock grid of the drilling model was broken (Fig. 11) between 0.07 and $0.08 \mathrm{~s}$, in which the broken grids were distributed at the edge of the outer ring of the blade. The rock stress was at the maximum and the rock-breaking condition was reached. After the unit grids were broken, the stress on the upper surface of the rock decreased, in which the maximum stress was $63.87 \mathrm{MPa}$. The grid unit with the maximum stress could be found at the edge of the broken small rock pit. Moreover, the grid was greatly deformed, and the stress was large before the rock was broken. However, after the rock was broken, the stress markedly decreased. The stress of the rock increased after it began to come in contact with the rock, which could mean that the bit was likely damaged in the initial rock-breaking stage. Therefore, the WOB should be applied smoothly and slowly (i.e., excessive WOB should be avoided) at the initial drilling stage to avoid the bit damage caused by the sudden breaking of the rock.

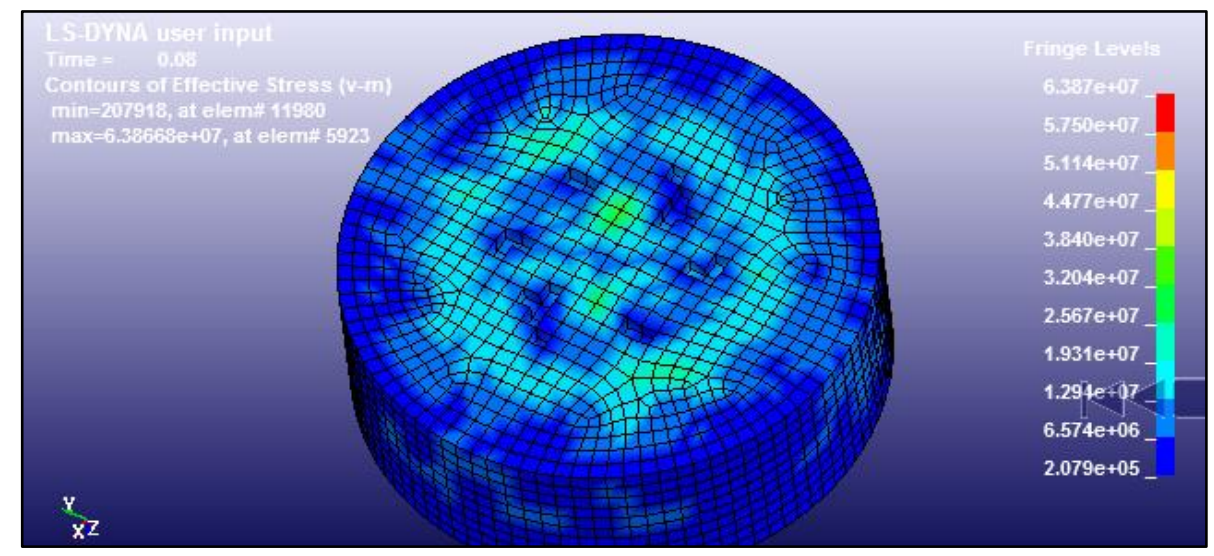

Figure 11: Stress at the moment when the rock was broken by the bit.

\subsection{Dynamic stress analysis in the rock-breaking stage of normal drilling}

The stress of rock changed with the continuous drilling of the bit. After the drilling reached $0.1 \mathrm{~s}$, the maximum dynamic stress value was only $66.3 \mathrm{MPa}$ in the normal drilling process. 
After the bit was drilled for $0.35 \mathrm{~s}$, the maximum dynamic stress increased to $85 \mathrm{MPa}$. After the drilling reached $0.6 \mathrm{~s}$, some parts of the rock were broken anew, and the maximum dynamic stress decreased to $70.8 \mathrm{MPa}$. The dynamic stress increased to $99.6 \mathrm{MPa}$ at $0.86 \mathrm{~s}$ after the rock was broken again (Fig. 12). After the initial rock breaking, the maximum dynamic stress fluctuated with the continuous breaking of the rock, and the maximum fluctuation range was 66.3-99.6 MPa, which was much lower than that at the initial rock-breaking stage. The nephogram of the rock stress during drilling (Fig. 12) exhibited the same relationship as that between rock-breaking load and time (Fig. 9).

The stress nephogram shown in Fig. 12 demonstrates the fluctuation to a certain extent of the dynamic stress of rock breaking during normal drilling. However, the fluctuation range was smaller than the initial dynamic stress. The force exerted by the rock on the bit was stable. Therefore, we can infer that the bit has been operated stably during normal drilling and is unlikely to be damaged, which corresponds to a normal wear stage.

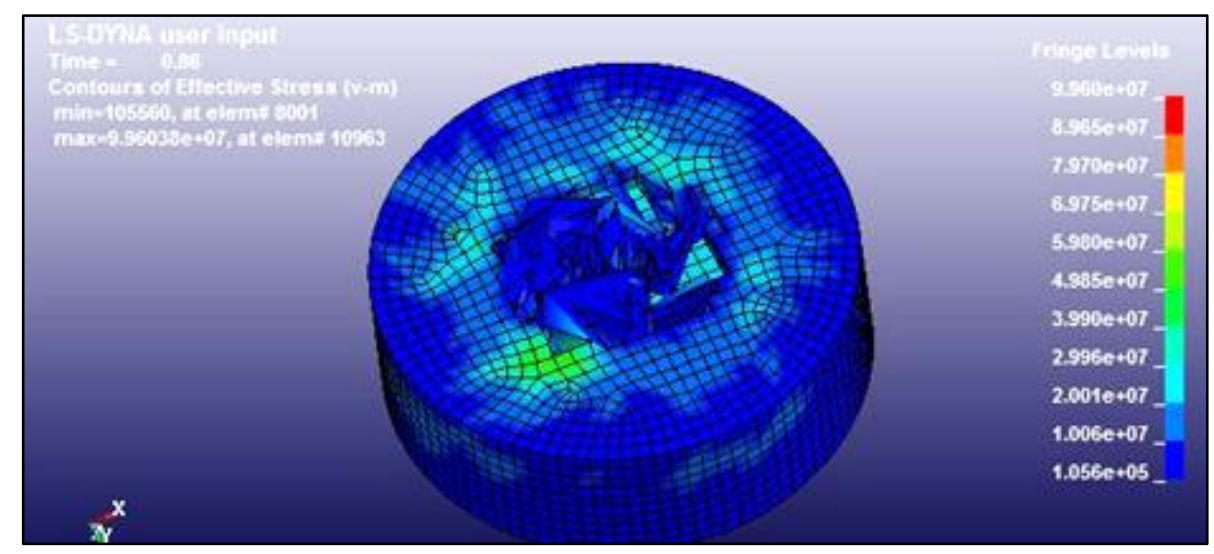

Figure 12: Rock stress during bit drilling.

\subsection{Analysis of the rock-breaking process}

The simulation results of rock breaking by the bit indicate that the rock grids at the edge of the bit blade were first broken, followed by the grids near the broken grid. Eventually, the grids located directly under the bit blade were completely damaged, that is, the breaking pit was enlarged. The rock grids located directly under the bit blade were severely deformed during bit rotation (Fig. 13). At $0.4 \mathrm{~s}$, the rock grid positioned directly under the bit blade was completely broken, and the grids under the cylindrical hole of the bit were also deformed. However, the rock grids were only severely deformed, but they were not broken.

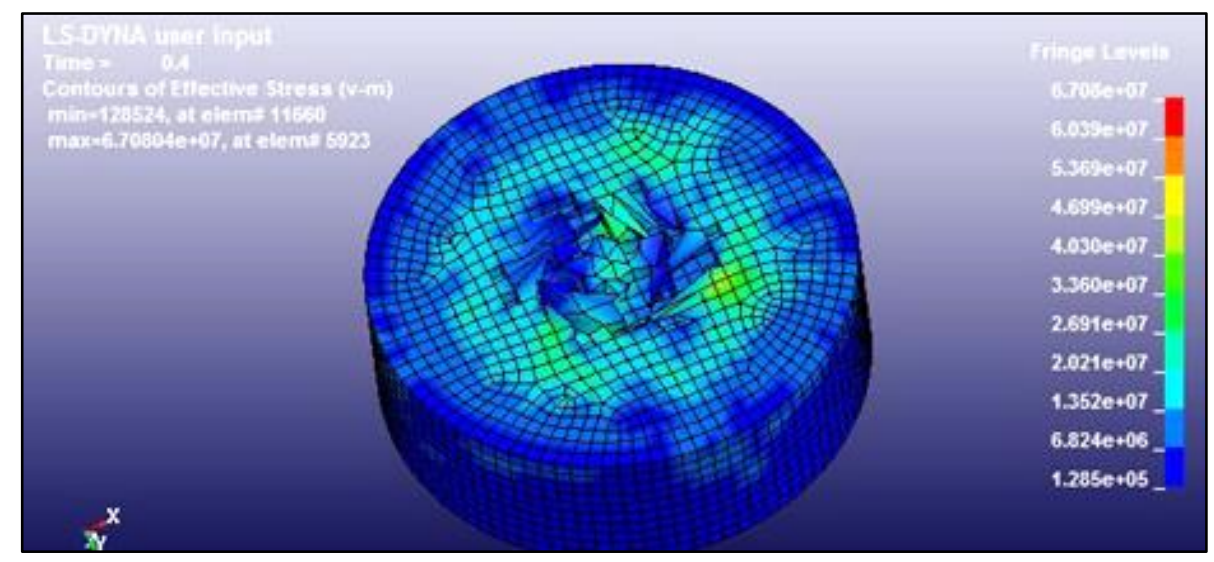

Figure 13: Rock-breaking expansion process. 
The rock-breaking model can be divided into three areas, namely, the rock pit area, damaged unit area, and undamaged unit area, as shown in Fig. 14. The rock pit area is the breaking pit of the rock. The damaged unit area is distributed around the breaking pit. The grids of the undamaged unit area remain in the linear elastic stage, and the rock in this area is not broken. The position of the rock pit area is related to the spatial position of the blade edge, and the size of rock pit area influences the rock-breaking efficiency of the damaged area. At a certain range, the larger is the broken area, the easier it is to break the rock grid unit at the edge of the broken pit. This phenomenon indicates a relatively good spatial position relationship between the blade and the cutters. Therefore, in view of improving the rock-breaking efficiency of the bit, the influence of spatial position on the distribution and size of the breaking pits should be considered when designing the bit blade and the cutter placement.

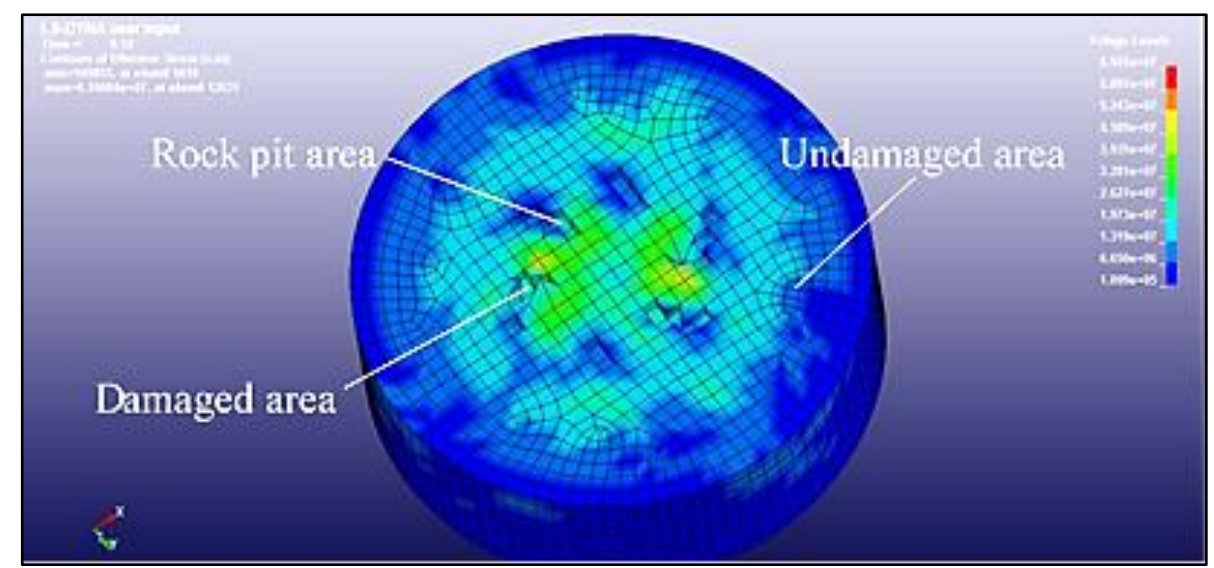

Figure 14: Rock-breaking area.

\section{CONCLUSION}

This study established a drilling model. The dynamic stress of the rock was calculated and analysed by the finite element method. The following conclusions can be drawn:

(1) The design cycle of a bit can be effectively shortened by simulating the drilling and analysing the dynamic stress of rock.

(2) The influence of the relative position of the bit blade and cutter on rock-breaking efficiency can be determined by simulating the dynamic rock-breaking process.

(3) The two obvious stages in the rock-breaking process by a bit are the initial rock-breaking stage and the rock-breaking stage during normal drilling. The dynamic rock-breaking stress in the normal drilling stage can vary from 66.3 to $99.6 \mathrm{MPa}$, a value range lower than $278.4 \mathrm{MPa}$ in the initial rock-breaking stage.

(4) The laboratory experiments carried out in this study verified the correctness of the simulation results. In the laboratory drilling process, the axial force and the tangential force of the spudding bit were 1.85 and $1.60 \mathrm{kN}$, respectively. During normal drilling, the axial force and the tangential force of the bit varied from 0.2 to $0.9 \mathrm{kN}$ and from 0.15 to $0.6 \mathrm{kN}$, respectively. The change in test load was consistent with that of dynamic stress.

(5) The load and the dynamic stress during normal drilling are lower than those during spudding. The bit is less likely to be damaged during spudding. During normal drilling, the bit is normally worn.

By analysing the dynamic rock breaking criterion, this study can economically and efficiently simulate and calculate the dynamic stress of rock by using FEM. The approach could quickly and efficiently design the relative positions of the PDC bit blades and cutters. The findings offer important reference value in designing PDC bits and improving drilling efficiency. 
Yu, Zou, Zhang: Analysis of Rock Dynamic Stresses During the Drilling by Polycristallyne ...

\section{REFERENCES}

[1] Zhang, J. Z. (2013). Study and Practice of Drilling Technology, China University of Mining and Technology Press, Beijing (in Chinese)

[2] Pryhorovska, T. O.; Chaplinskiy, S. S.; Kudriavtsev, I. O. (2015). Finite element modelling of rock mass cutting by cutters for PDC drill bits, Petroleum Exploration and Development, Vol. 42, No. 6, 888-892, doi:10.1016/S1876-3804(15)30087-2

[3] Jing, Y. H.; Yuan, X. W.; Jiang, L.; Zhang, H.; Ni, H. J. (2019). Numerical simulation and field experimental study on rock breaking in high speed rotating percussion drilling, Journal of China University of Petroleum, Vol. 43. No. 1, 75-80, doi:10.3969/j.issn.1673-5005.2019.01.009

[4] Hu, H. G.; Guan, Z. H.; Zhang, B.; Xu, Y. Q.; Liu, Y. W.; Wang, B. (2021). Structure design of weight-on-bit self-adjusting PDC bit based on stress field analysis and experiment evaluation, Journal of Petroleum Science and Engineering, Vol. 196, Paper 107692, 15 pages, doi:10.1016/j.petrol.2020.107692

[5] Wan, Q.; Zheng, M. L.; Yang, S. C.; Sun, J. K. (2019). Optimization of micro-texture distribution through finite-element simulation, International Journal of Simulation Modelling, Vol. 18, No. 3, 543-554, doi:10.2507/IJSIMM18(3)CO15

[6] Artola, L. G.; Arriola, P. J. A.; de Buruaga, M. S.; Soler, D.; de Zarate, G. O.; Aizpuru, O.; Mielgo, R. (2019). Finite element modelling of the turning of quenched steels, DYNA, Vol. 94, No. 3, 331337, doi: $10.6036 / 8846$

[7] Ahmadi, M.; Seyedin, S. H.; Seyedin, S. V. (2019). Investigation of the mechanical performance of fiber-modified ceramic composites using finite element method, Tehnicki glasnik-Technical Journal, Vol. 13, No. 3, 173-179, doi:10.31803/tg-20181006143504

[8] Miyazaki, K.; Ohno, T.; Karasawa, H.; Imaizumi, H. (2019). Performance of polycrystalline diamond compact bit based on laboratory tests assuming geothermal well drilling, Geothermics, Vol. 80, 185-194, doi:10.1016/j.geothermics.2019.03.006

[9] Mazen, A. Z.; Mujtaba, I. M.; Hassanpour, A.; Rahmanian, N. (2020). Mathematical modelling of performance and wear prediction of PDC drill bits: impact of bit profile, bit hydraulic, and rock strength, Journal of Petroleum Science and Engineering, Vol. 188, Paper 106849, 18 pages, doi:10.1016/j.petrol.2019.106849

[10] Agostini, C. E.; Sampaio, M. A. (2020). Probabilistic neural network with Bayesian-based, spectral torque imaging and deep convolutional auto-encoder for PDC bit wear monitoring, Journal of Petroleum Science and Engineering, Vol. 193, Paper 107434, 33 pages, doi:10.1016/ j.petrol.2020.107434

[11] Abbas, R. K.; Musa, K. M. (2019). Using Raman shift and FT-IR spectra as quality indices of oil bit PDC cutters, Petroleum, Vol. 5, No. 3, 329-334, doi:10.1016/j.petlm.2018.10.003

[12] Agawani, W.; Al-Enezi, D. R.; Pandya, M.; Gupta, P.; Abdelhamid, A.; Al-Habib, H.; El-Touny, S.; Ahmed, T.; Mohammed, J. A. (2018). Engineered hybrid drill bit technology doubles drilling performance in impact-prone carbonates heavy oil application, Proceedings of the 2018 SPE International Heavy Oil Conference and Exhibition, Paper SPE-193704-MS, 14 pages, doi:10.2118/193704-MS

[13] Abbas, R. K. (2018). A review on the wear of oil drill bits (conventional and the state of the art approaches for wear reduction and quantification), Engineering Failure Analysis, Vol. 90, 554584, doi:10.1016/j.engfailanal.2018.03.026

[14] Saksala, T.; Gomon, D.; Hokka, M.; Kuokkala, V.-T. (2014). Numerical and experimental study of percussive drilling with a triple-button bit on Kuru granite, International Journal of Impact Engineering, Vol. 72, 56-66, doi:10.1016/j.ijimpeng.2014.05.006

[15] Wang, B.; Li, J.; Zou, D. Y.; Yang, H. W.; Wang, K. (2018). Design and application of a PDC hybrid drill bit with impregnated diamond insert for the hard formation with strong abrasivity, Special Oil \& Gas Reservoirs, Vol. 25, No. 1, 169-174, doi:10.3969/j.issn.1006-6535.2018.01.035

[16] Wang, X.; Liu, Z. Y.; Qu, S. N.; Su, C. (2018). Research on rock breaking simulation of PDC bit under rock property parameters in Weibei oilfield, China Energy and Environmental Protection, Vol. 40, No. 9, 99-103, doi:10.19389/j.cnki.1003-0506.2018.09.020 
[17] Zou, D. Y.; Xu, C. K.; Yi, Y.; Chen, X. P.; Yu, J. P. (2017). An experimental study on PDC bits' cutter parameters and formation adaptability, Natural Gas Industry, Vol. 37, No. 9, 85-90, doi:10.3787/j.issn.1000-0976.2017.09.011

[18] Niu, S. W.; Zheng, H. L.; Yang, Y. X.; Chen, L. (2018). Experimental study on the rock-breaking mechanism of disc-like hybrid bit, Journal of Petroleum Science and Engineering, Vol. 161, 541550, doi:10.1016/j.petrol.2017.12.011

[19] Han, L. J.; Wang, Z. G.; Feng, G. T.; Wei, Z. (2013). Petroleum Rock Mechanics: Drilling Operations and Well Design, China University of Petroleum Press, Beijing

[20] Liu, Y. Q.; Yu, G. J. (2018). Elasticity Mechanics for Engineering, China University of Petroleum Press, Beijing

[21] Macyszyn, L.; Jedryczka, C.; Staniek, R. (2019). Design and finite element analysis of novel twostage magnetic precession gear, International Journal of Simulation Modelling, Vol. 18, No. 4, 586-595, doi:10.2507/IJSIMM18(4)487

[22] Luo, P. P.; Wang, S. R.; Hagan, P.; Huang, Q. X.; Cao, C.; Gamage, K. (2019). Mechanical performances of cement-gypsum composite material containing a weak interlayer with different angles, DYNA, Vol. 94, No. 4, 447-454, doi:10.6036/9191

[23] Zhang, S. R.; Song, R.; Wang, C.; Shang, C.; Wei, P. Y. (2019). Modification of a dynamic constitutive model-HJC model for roller-compacted concrete and numerical verification, Journal of Vibration and Shock, Vol. 38, No. 12, 25-31, doi:10.13465/j.cnki.jvs.2019.12.004 